\title{
Two-Channel Optoelectronic Sensor Employing Cavity Enhanced Absorption Spectroscopy
}

\begin{abstract}
J. WOJTAS*
Institute of Optoelectronics, Military University of Technology, S. Kaliskiego 2, 00-908 Warsaw, Poland

In the paper, a description of two-channel of optoelectronic sensor basing on cavity enhanced absorption spectroscopy is presented. In contrast to the typical cavity enhanced absorption spectroscopy systems, here a special optical system is applied. It provides possibility to direct more than one beam of laser radiation into a cavity. Thus, special lasers controller system and photoreceiver were developed as well. The setup includes two pulsed laser diodes. The optical signals from the lasers are registered with one special constructed photoreceiver, which is characterized by wide range of the detected wavelengths. Moreover, there was applied time division multiplexing technique, well-known in the telecommunication systems. Every laser is assigned to the suitable channel - the strictly determined temporary window. In this window, the signal from the exits of the optical cavity is registered. In the system, optical signal from many laser sources can be measured and value of absorption coefficient at a few different wavelengths can be determined parallel. Typical cavity enhanced absorption spectroscopy system is designed to measure only one gas concentration, while the developed setup provides possibility to detect a trace concentration of two gases at the same time.
\end{abstract}

PACS: 07.07.Df, 42.25.Dd, 42.55.Px, 42.60.Da, 42.62.Fi

\section{Introduction}

There are many methods used for gases detection. In general, they can be divided for remote methods (standoff) and in situ methods that are used exactly in the place of occurring gas. Former contain passive methods, which apply optical radiation emitted by a thermal object and active ones, which are more frequently used in remote gas detection application. The latter are very popular. They can be divided into spectroscopic and non-spectroscopic methods. For example, widely used chemical methods belong to the first group. They are based on the use of certain chemical reactions, which may indicate the presence of the substance sought [1]. In the second, methods applying optical phenomena like scattering, emission and absorption can be listed. In all spectroscopic methods the properties of the sample are determined on the basis of the measured spectral characteristics of radiation [2]. Absorption spectra can be defined as the set of all electron crossings from lower energy levels to higher ones. They cause an increase in molecules energy. In case of the emission spectra there is inverse situation. The spectra correspond to the reduction of molecules energy as a result of electrons transitions from higher energy levels to lower ones. Scattering spectra rely on a change in the frequency spectra diffuse radiation in relation to the frequency of incident radiation, due to the partial change of the photon energy as a result of impact with

* e-mail: jwojtas@wat.edu.pl the molecules. However, in this case there is no effect of radiation absorption or emission [1,3].

Cavity enhanced absorption spectroscopy (CEAS) belongs to one of the most sensitive laser absorption spectroscopy's techniques. It was developed in 1998 by Engel [4]. CEAS is based on injection a beam of radiation under the very small angle $(\varphi)$ in relation to the optical axis of cavity (i.e. off axis). The radiation is reflected inside the optical cavity similarly as inside multipass cell (Fig. 1). Therefore, dense structure modes are received. The weak modes structure of the resonance cavity causes that the all system has the small sensibility on the changes of the cavity and the laser frequency [5]. Sensors using CEAS method attain sensitivity amounting about $10^{-9} \mathrm{~cm}^{-1}[6,7]$.

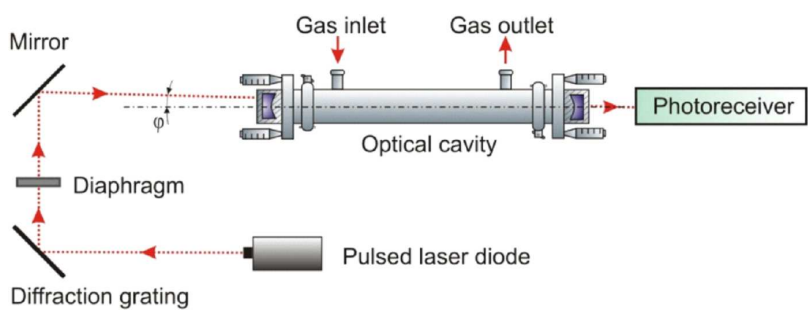

Fig. 1. Idea of CEAS setup.

A pulse of optical radiation is injected into the cavity through one of the mirrors. Then inside the cavity multiple reflections occur. After each reflection, part of the radiation exiting from the cavity is registered with 
the photoreceiver. The output signal from the photoreceiver is proportional to the intensity of radiation propagating inside the optical cavity. If the laser wavelength is matched to the absorption spectra of gas filling the cavity, the cavity quality decreases. Thus, parameters of the signal from the photoreceiver are changed. Thanks to this, the absorption coefficient and concentration of gas can be determined [8].

Typical CEAS setup provides possibility of investigation of only one gas absorption line. Generally it consists of a pulse radiation source, optical cavity, photoreceiver, and a signal processing system $[9,10]$. In the experimental setup two lasers and two optical channels were used. Such construction makes it possible to detect a trace concentration of two gases with different absorption spectra at the same time.

\section{Experimental setup}

The experimental setup is presented in Fig. 2. As radiation sources two laser diodes were applied. In the first channel, $635 \mathrm{~nm}$ wavelength $\mathrm{AlGaInP}$ laser diode was used (type DL-5038-031, Sanyo). The second channel was equipped with violet laser diode (type GH04125A2A,
Sharp). Its emission wavelength was matched to the nitrogen dioxide absorption spectrum.

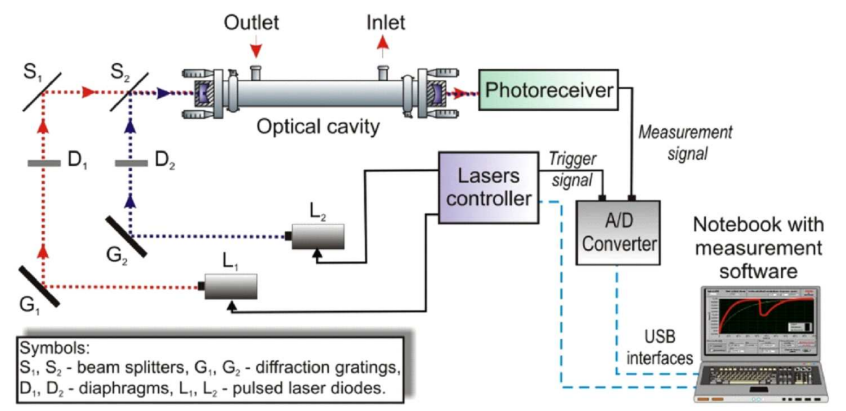

Fig. 2. Experimental setup.

In the wavelength region of the first laser there are no significant absorption spectra of other atmosphere gases [11]. Such configuration provides possibility of simultaneous nitrogen dioxide concentration measurements and optical system controlling. The main parameters of the lasers are presented in Table.

Parameters of the applied laser diodes.

TABLE

\begin{tabular}{c|c|c|c|c|c|c}
\hline \hline $\begin{array}{c}\text { Laser } \\
\text { type }\end{array}$ & $\begin{array}{c}\text { Lasing } \\
\text { wavelength } \\
{[\mathrm{nm}]}\end{array}$ & $\begin{array}{c}\text { Threshold } \\
\text { current } \\
{[\mathrm{mA}]}\end{array}$ & $\begin{array}{c}\text { Operating } \\
\text { voltage } \\
{[\mathrm{V}]}\end{array}$ & $\begin{array}{c}\text { Optical } \\
\text { power } \\
{[\mathrm{mW}]}\end{array}$ & $\begin{array}{c}\text { Perpendicular } \\
\text { divergence } \\
{\left[{ }^{\circ}\right]}\end{array}$ & $\begin{array}{c}\text { Parallel } \\
\text { divergence } \\
{\left[{ }^{\circ}\right]}\end{array}$ \\
\hline DL-5038 & $635-645$ & $90-110$ & $2.4-2.7$ & 35 & $25-35$ & $6-9^{\circ}$ \\
GH04125A2A & $400-413$ & $125-150$ & $5.4-6.4$ & 150 & $16-25$ & $6-12^{\circ}$
\end{tabular}

The lasers beams were formed with diffraction gratings and diaphragms, and they were directed with beam splitters (CVI Melles Griot) into the optical cavity. The transmission characteristics of the beam splitters are presented in Fig. 3. The optical cavity was built of two spherical mirrors, the reflectivities of which reach value of 0.998 and 0.999 at the wavelength of interest (Fig. 3). The distance between the mirrors was $50 \mathrm{~cm}$. Sensitivity of the first channel of the sensor was $2 \times 10^{-6} \mathrm{~cm}^{-1}$ and of the second one reach value of $10^{-6} \mathrm{~cm}^{-1}$ (Fig. 4).

The optical signal from the cavity was registered with a photomultiplier (type R7518, Hammamatsu). It is characterized by high gain $\left(1.1 \times 10^{7}\right)$, high speed and low dark current. Because of photomultiplier (PMT) high resistance, a transimpedance preamplifier was used to amplify signal from PMT [12]. Next, a signal from the preamplifier was digitized with $100 \mathrm{MS} / \mathrm{s}$ sampling rate. Data from analogue-to-digital (A/D) converter were transmitted to a computer through USB interface. There was developed special software, which provides

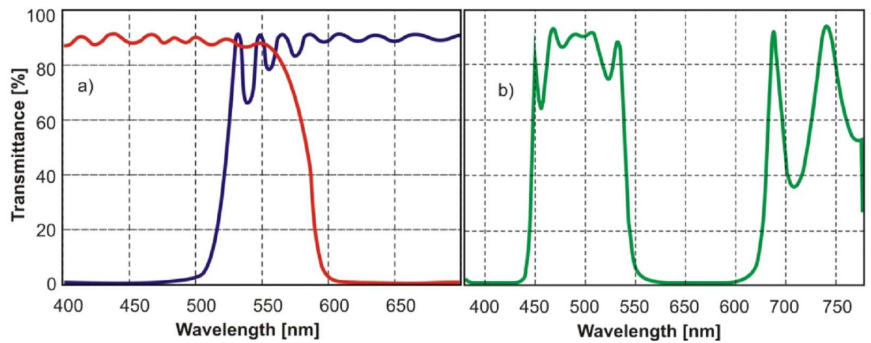

Fig. 3. Transmittance characteristics of the beam splitters (a) and cavity mirrors (b).

control of the lasers operating, measuring process and data processing.

Using the specially constructed photoreceiver the optical signals from the lasers are registered. Each of the lasers is assigned to the suitable measurement channel the strictly determined temporary window. In this window, the signal from the exits of the optical cavity is reg- 


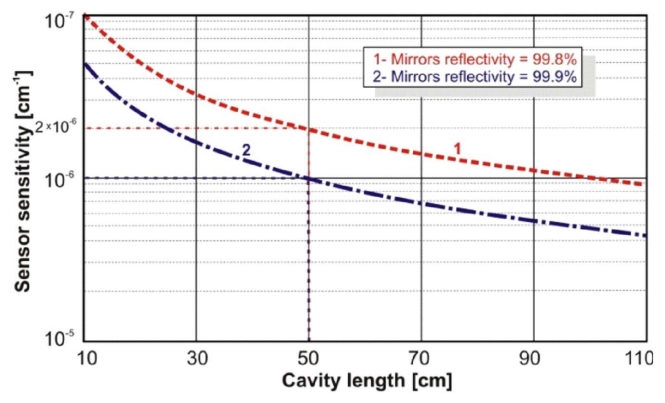

Fig. 4. Dependence of sensor sensitivity on the cavity length and the mirrors reflectivity.

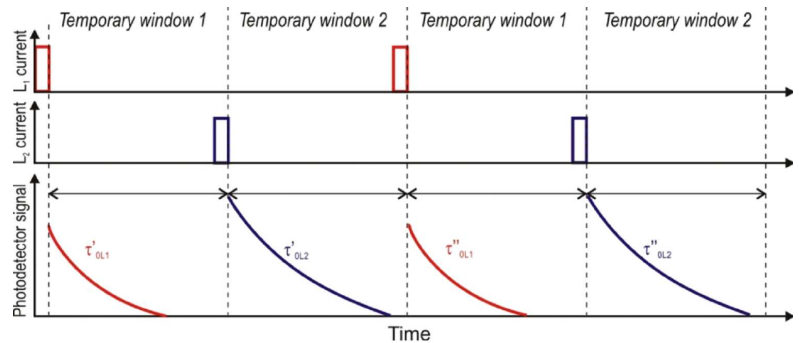

Fig. 5. Example of lasers control signals and photoreceiver signal registered with the oscilloscope.

istered (Fig. 5). This is similar to time division multiplexing technique used in the telecommunication systems.

In the cavity absorption spectroscopy the gas concentration (cavity quality) can be determined on the basis of decay time measurements, by measuring the phase shift and by measuring the signal amplitude $[10,13]$. In the experiments first method was applied. Determination of the concentration of the examined gas is a two-step process. Firstly, measurement of the signal decay time $\left(\tau_{0}\right)$ in the optical cavity not containing the absorber (tested gas) is performed. Secondly, measuring the signal decay time $\tau$ in the cavity filled with the tested gas is carried out. Knowing the absorption cross-section $(\sigma)$ of the examined gas, its concentration can be calculated from the formula

$$
C=\frac{1}{C_{0} \sigma c}\left(\frac{1}{\tau}-\frac{1}{\tau_{0}}\right) \times 10^{9}[\mathrm{ppb}]
$$

where $C_{0}$ denotes the Loschmidt number, $c$ - the light speed, $\sigma$ - absorption cross-section for nitrogen dioxide at the wavelength of interest $[14,15]$.

\section{Experimental results}

During experiments three stages of measurements were conducted. First, $100 \mathrm{ppb}$ of nitrogen dioxide mixture was delivered to the sensor. Second, measurements were carried out for cavity filled the different $\mathrm{NO}_{2}$ concentrations (from 100 to $345 \mathrm{ppb}$ ). In the last stage of researches, $100 \mathrm{ppb}$ of nitrogen dioxide humid mixture was used. The relative humidity of the gas samples was $80 \%$.
Investigated gases were delivered to the cavity from modular gas standards generator type 491M equipped with humidification gas module HG type (Kin-Tek). The generator assures automatic system clearance and the sensor calibrations with use of inert gas. Example of research results are presented in Figs. 6, 7, and 8.

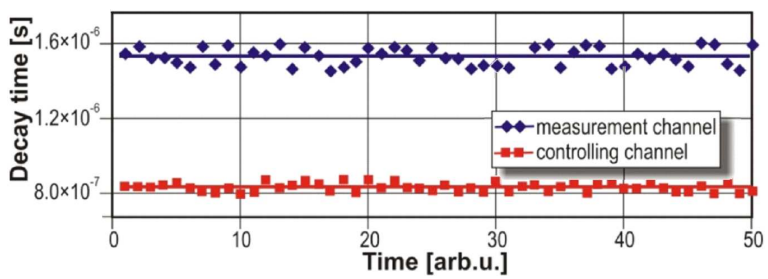

Fig. 6. Results of $100 \mathrm{ppb} \mathrm{NO}_{2}$ concentration measurements.

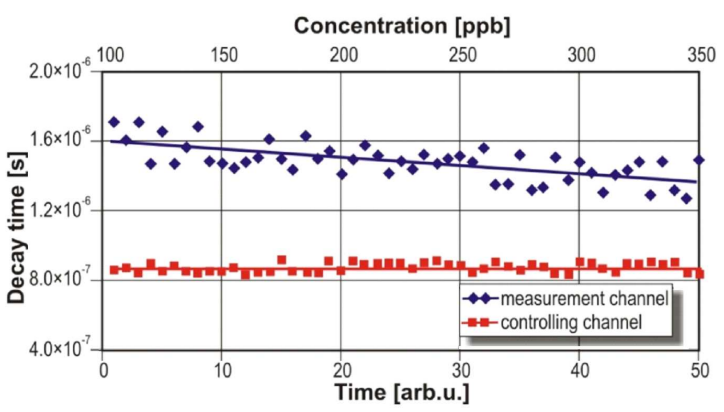

Fig. 7. Results of different $\mathrm{NO}_{2}$ concentrations measurements.

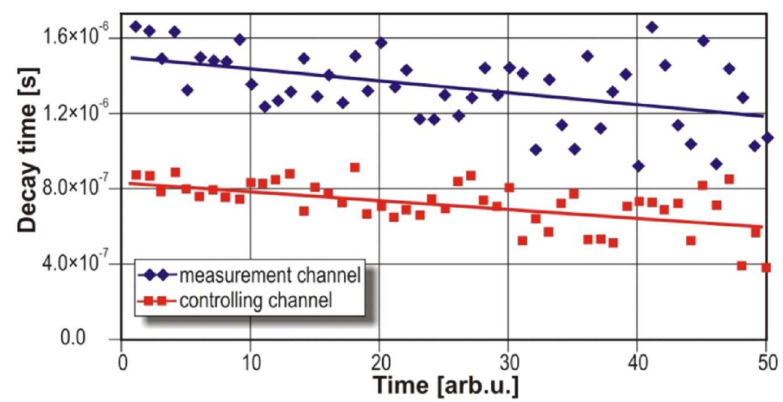

Fig. 8. Results of measurements with $100 \mathrm{ppb} \mathrm{NO}_{2}$ humid mixture $(\mathrm{RH}=80 \%)$.

The measurements of decay time were carried out in both channel simultaneously. When the cavity was filled with $100 \mathrm{ppb} \mathrm{NO}_{2}$ dry mixture, the decay times in controlling channel and measurement one were almost constant, and their mean values were $0.83 \mu \mathrm{s}$ and $1.53 \mu \mathrm{s}$, respectively (Fig. 6).

While the concentration of the nitrogen dioxide was increased, decay time in the controlling channel was still almost constant, but in the second channel the decay time decreased proportional to the $\mathrm{NO}_{2}$ concentration (Fig. 7). 
When humid mixture of $100 \mathrm{ppb} \mathrm{NO}_{2}$ was delivered to the sensor, both decay times were decreased (Fig. 8). It was caused by moisture, which distorted cavity mirrors reflectance. Thus, reason of signal decrease in measurement channel can be identified thanks to controlling channel. If nitrogen dioxide concentration is increased, only decay time measured in the channel with violet laser is decreased.

When the uncertainty of decay time measurements does not exceed $3 \%$, thus nitrogen dioxide sensor detection limit attains the value of $30 \mathrm{ppb}$. It was calculated from the formula

$$
C_{L}=\frac{1}{\tau_{0} \sigma c} \delta_{\tau}=\frac{(1-R)}{\sigma L} \delta_{\tau},
$$

where $\delta_{\tau}$ is the relative uncertainty of the decay time measurement, which can be described as

$$
\delta_{\tau}=\frac{\tau_{0}-\tau_{L}}{\tau_{0}} 100 \%,
$$

where $\tau_{L}$ denotes a decay time of the optical cavity for minimal absorber concentration.

\section{Summary}

The main reason for developing more perfect sensors detecting various chemical compounds is ensuring safety, e.g. in the vicinity of factories, in important objects like airports, in environmental protection, health care, etc. These applications have a significant impact on the performance of sensors.

Presented sensor provides possibility of simultaneous nitrogen dioxide concentration measurements and cavity quality controlling. The controlling process is performed continuously by the use of the second channel outside the absorption spectrum of investigated gas. The sensitivity of the nitrogen dioxide sensor attains value of $30 \mathrm{ppb}$. Moreover, developed sensor is able to detect trace concentration of two gases at the same time. Then the both channel should be appropriately matched to absorption spectrum of the investigated gases.

The presented setup is of the greatest interest with respect to the possible applications. For example, it can be used in environment monitoring, luggage monitoring in ports, on airports, entry points, as well as in strategic objects and rooms also in undertakings connected with the counteraction to terrorist attacks. The sensor can be used during different measurement conditions. Any in- correct results of concentration measurements caused by the sensor malfunction can be identified and discarded through the use of cavity parameters monitoring channel.

\section{Acknowledgments}

The project was financed by the Polish Ministry of Science and Higher Education (O N515 115836).

\section{References}

[1] M.W. Sigrist, Air Monitoring by Spectroscopic Techniques, Wiley, New York 1994.

[2] A.F. Lagalante, Appl. Spectrosc. Rev. 34, 173 (1999).

[3] B.E.A. Saleh, M.C. Teich, Fundamentals of Photonics, 2nd ed., Wiley, New York 2007.

[4] R. Engeln, G. Berden, R. Peeters, G. Meijer, Rev. Sci. Instrum. 69, 3763 (1998).

[5] J.B. Paul, L. Lapson, J.G. Anderson, Appl. Opt. 40, 4904 (2001).

[6] V.L. Kasyutich, C.S.E. Bale, C.E. Canosa-Mas, C. Pfrang, S. Vaughan, R.P. Wayne, Appl. Phys. B 76, 691 (2003).

[7] G. Berden, R. Peeters, G. Meijer, Int. Rev. Phys. Chem. 19, 565 (2000).

[8] I. Courtillot, J. Morville, I. Motto-Ros, D. Romanini, Appl. Phys. B 85, 407 (2006).

[9] G. Berden, R. Engeln, Cavity Ring-Down Spectroscopy: Techniques and Applications, WileyBlackwell, Chichester 2009.

[10] K.W. Busch, M.A. Busch, Cavity-Ringdown Spectroscopy, An Ultratrace-Absorption Measurement Technique, ACS Symp. series 720, Washington DC 1999, p. 10.

[11] Hitran 2008 database, Ontar Corporation, www. ontar.com .

[12] J. Wojtas, Z. Bielecki, Opto-Electron. Rev. 16, 44 (2008).

[13] J. Wojtas, A. Czyżewski, T. Stacewicz, Z. Bielecki, J. Mikolajczyk, Proc. SPIE 5954, 174 (2005).

[14] J. Wojtas, A. Czyzewski, T. Stacewicz, Z. Bielecki, Opt. Appl. 36, 461 (2006).

[15] M. Nowakowski, J. Wojtas, Z. Bielecki, J. Mikołajczyk, Acta Phys. Pol. A 116, 363 (2009). 